Section Editor Mitchell S.V. Elkind, MD, MS

Sailaja Enduri, MD

Matthew R.G. Taylor, $\mathrm{MD}, \mathrm{PhD}$

Teerin Liewluck, MD

Correspondence to

Dr. Liewluck:

teerin.liewluck@ucdenver.edu

Clinical Reasoning:

\title{
A 52-year-old woman with progressive proximal weakness
}

\section{SECTION 1}

A 52-year-old woman presented with a 12-year history of progressive limb weakness. She first had trouble climbing stairs and later difficulty getting up from a chair. She also experienced hip and shoulder pain. Over the previous 2 years, combing her hair became difficult. She denied any eyelid drooping, double vision, chewing or swallowing difficulty, slurred speech, shortness of breath, numbness, tingling, autonomic symptoms, or cognitive problems. She did not have any rashes or joint pain. She had no other medical problems and was not taking any medications. She was born full-term and had normal developmental milestones. She was able to keep up with her peers and was a good runner during her childhood. The patient was of German descent and her father and paternal uncle developed leg weakness in their early 40s and were diagnosed with limb-girdle muscular dystrophy (LGMD). They died in a plane crash in their late 40s. The patient's older brother and her uncle's son developed leg weakness in their early $40 \mathrm{~s}$ and were also diagnosed with LGMD. Both died in their mid-to-late 50s due to respiratory failure. Her brother also had peripheral neuropathy. The diagnosis of LGMD in all family members is based on clinical presentations and muscle biopsies. Genetic studies were not performed. There was no family history of dementia, bone disorder, or motor neuron disease. Her neurologic examination revealed symmetrical weakness of shoulder and hip girdle muscles (Medical Research Council [MRC] grade 4), as well as anterior leg compartment muscles (MRC grade 4+). She also had scapular winging bilaterally. There was no myotonia, muscle rippling, fasciculation, muscle atrophy, or hypertrophy. Deep tendon reflexes were normal except for absent ankle reflexes on both sides. Cognition, cranial nerves, sensory examination, and coordination were normal. She had high-arched feet and hammertoes. There was neither joint contracture nor spinal rigidity.

\section{Questions for consideration:}

1. What is your differential diagnosis at this point?

2. What testing would be helpful to narrow the differential diagnosis? 


\section{SECTION 2}

This patient presented with several years of progressive symmetrical proximal limb weakness without sensory, ocular, or bulbar symptoms. The pure motor syndrome could be due to disorders affecting motor neurons, motor nerves, neuromuscular junctions, or muscles. The diagnosis of LGMD in her father, uncle, brother, and cousin points toward autosomal dominant disorder of skeletal muscle. The symmetrical proximal weakness is the most common weakness pattern of hereditary myopathy or muscular dystrophy. These patients typically have normal reflexes, but the ankle reflexes could be diminished or absent in patients with superimposed peripheral neuropathy, severe distal myopathic weakness, or S1 radiculopathy.

Hereditary disorders of motor neurons include familial amyotrophic lateral sclerosis (ALS), spinal muscular atrophy (SMA), and spinobulbar muscular atrophy (SBMA). Most variants of familial ALS and a few variants of SMA are inherited in an autosomal dominant manner. Patients with ALS typically present with asymmetric distal weakness associated with mixed upper and lower motor neuron findings, whereas patients with SMA develop symmetric proximal weakness associated with lower motor neuron features. SBMA is an X-linked lower motor neuron disease. Manifesting carriers of SBMA have been reported, but symptoms were much milder than what was observed in affected male patients. The absence of fasciculation and muscle atrophy after several years of weakness make familial ALS, SMA, and SBMA unlikely. Hereditary motor neuropathies are a group of autosomal dominant or less commonly autosomal recessive motor neuropathies with preferential distal weakness and sparing of proximal muscles. Hereditary disorders of neuromuscular transmission or congenital myasthenic syndromes are inherited in an autosomal recessive fashion except for the slowchannel congenital myasthenic syndrome, which is an autosomal dominant disorder. Patients with slow-channel congenital myasthenic syndrome typically have not only ocular involvement, but also wrist or finger extensor weakness.

The patient was initially evaluated at our institute 10 years ago. She recently returned for a follow-up visit due to the concern of developing respiratory compromise. All these results were from her initial evaluation. Serum creatine kinase (CK) level was 539 U/L (normal <195 U/L). Sural sensory nerve action potential, peroneal compound muscle action potential (CMAP), and tibial CMAP had mildly slow conduction velocities with normal amplitudes and distal latencies. At that time, the record showed the patient did not have ankle dorsiflexor weakness and the ankle reflexes were intact. A needle EMG revealed small and rapidly recruiting motor unit potentials associated with fibrillation potentials in deltoid, biceps, iliopsoas, and gastrocnemius. There were no myotonic discharges. Her EKG showed normal sinus rhythm and suggested left atrial enlargement. Echocardiogram was not performed.

\section{Questions for consideration:}

1. Based on these findings, what is your current differential diagnosis?

2. What further evaluation would be helpful? 


\section{SECTION 3}

The mildly elevated CK level can be seen in either myopathy or motor neuron disease, but the small and rapidly recruiting motor unit potentials are consistent with the myopathic process. These needle EMG features and the absence of ptosis or ophthalmoparesis point away from neuromuscular junction disorders. Given the family history of LGMD and the longstanding history of muscle weakness, it is unlikely that the patient has acquired myopathy (e.g., inflammatory myopathy). The differential diagnosis of autosomal dominant myopathy/muscular dystrophy includes LGMD1, myotonic dystrophy (DM1 and DM2), facioscapulohumeral dystrophy (FSHD), oculopharyngeal muscular dystrophy (OPMD), myofibrillar myopathy (MFM), autosomal dominant Emery-Dreifuss muscular dystrophy (EDMD), autosomal dominant inclusion body myopathy, mitochondrial myopathy due to nuclear DNA mutations, and certain subtypes of congenital myopathy or distal myopathy.

The symmetric hip and shoulder girdle weakness with sparing of the ocular, facial, and bulbar muscles can be seen in LGMD1, DM2, MFM, inclusion body myopathy, and adult-onset congenital myopathy. Some of the LGMD1 subtypes are allelic to MFM. Scapular winging is not uncommon in patients with MFM, inclusion body myopathy due to
VCP mutations, and some congenital myopathies (hyaline body myopathy and reducing body myopathy). The absence of ptosis and dysphagia makes OPMD unlikely. The sparing of facial muscles is uncommon in FSHD. The lack of clinical and electrical myotonia points away from DM1, but both clinical and electrical myotonia could be subtle or absent in DM2. The absence of early contracture makes EDMD unlikely. The mildly elevated CK level in this patient is much lower than what is typically observed in patients with muscular dystrophies due to the sarcolemmal defects (e.g., dystrophinopathy).

The absence of ankle reflexes in this patient is out of proportion to the mild degree of ankle dorsiflexor weakness, which suggests concomitant peripheral neuropathy or S1 radiculopathy rather than distal myopathy. Mildly slowing sural responses noted in pervious nerve conduction studies favor superimposed peripheral neuropathy rather than S1 radiculopathy. A mild cardiac abnormality was noted on the patient's EKG. A constellation of myopathy, peripheral neuropathy, and cardiac involvement, as seen in this patient, has been reported in LGMD1A, LGMD1B/autosomal dominant EDMD (laminopathy), MFM, and autosomal mitochondrial myopathy. Overall, the pattern of weakness, the winged scapulae, and the possible concomitant neuropathy
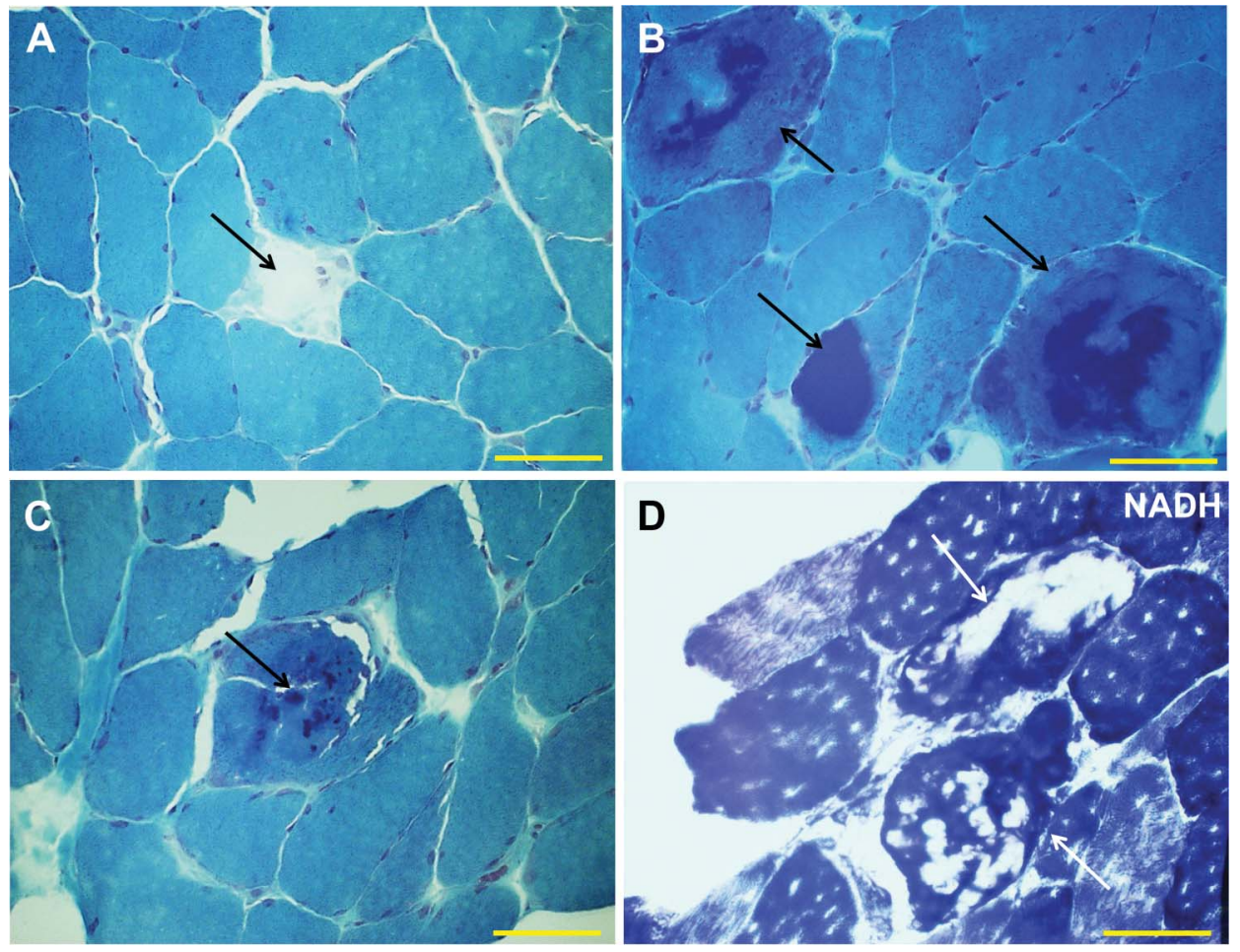

Modified Gomori trichrome stain demonstrates (A) a moderate variation in fiber size and scattered necrotic fibers (arrow); (B) a few fibers harboring amorphous hyaline deposits that are dark blue (arrows); (C) rare fibers containing cytoplasmic bodies (arrow). (D) On NADH dehydrogenase stain, the hyaline plaques are devoid of oxidative enzyme activity (arrows). Bar, 50 $\mu \mathrm{m}$. 
and cardiac involvement make MFM the most likely diagnosis. Moreover, the patient's brother and cousin died of respiratory failure and respiratory involvement occurs in some variants of MFM.

The patient had undergone left deltoid biopsy 10 years previously. Chronic noninflammatory myopathy was originally reported and the patient was diagnosed with LGMD1. Upon review of the biopsy slides, there were a few muscle fibers harboring characteristic features of MFM (figure).

\section{DISCUSSION}

MFM refers to a group of genetically heterogeneous muscular dystrophies with similar muscle biopsy findings (disintegration of myofibrils and aggregation of several proteins into distinct sarcoplasmic deposits), but diverse clinical phenotypes (limb-girdle, scapuloperoneal, distal, or axial weakness) and variable age at onset. Some of the LGMD1 subtypes are allelic to MFM. Cardiomyopathy and peripheral neuropathy are frequent associated features. ${ }^{1}$ EMG reveals myopathic motor unit potentials and abnormal irritability, often with myotonic discharges. ${ }^{1}$ Some patients have a combination of myopathic and neurogenic motor unit potentials or slow nerve conduction velocities. ${ }^{1}$ The causative genes are identified in only half of the patients, including MYOT (myotilin), ZASP (Z-band alternatively spliced PDZ-motif protein), DES (desmin), CRYAB ( $\alpha$ B-crystallin), FLNC (filamin C), BAG3 (BCL-2-associated athanogene-3), DNAJB6 (DNAJ/ HSP40 homolog, subfamily B, member 6), FHL1 (four-and-a-half-LIM protein 1), and TTN (titin). MYOT-MFM, DNAJB6-MFM, and DES-MFM are allelic to LGMD1. Respiratory involvement is common in patients with FLNC-MFM and TTN-MFM, and was reported in a few patients with DES-MFM, CRYAB-MFM, and MYOT-MFM. ${ }^{2,3}$ TTN-MFM patients usually develop respiratory insufficiency early in the disease course, ${ }^{3}$ whereas FLNC-MFM patients experience respiratory compromise later with disease progression. $^{2}$ The family history of respiratory compromise developing a decade after the onset of weakness as seen in our patient is typical of FLNC-MFM. Being of German descent also favors the diagnosis of FLNC-MFM, given that the founder FLNC mutation has been reported in German patients. ${ }^{3}$
FLNC sequencing in this patient showed a p.Trp2710X mutation. This p.Trp2710X mutation was first identified as the founder mutation in German patients and later was reported in patients from other ethnic backgrounds. ${ }^{3,4}$ FLNC encodes filamin $\mathrm{C}$, the muscle-specific protein that cross-links actin in the $\mathrm{Z}$-disc region and interacts with myotilin and sarcoglycans. ${ }^{4}$ Mutations in the rod domain of filamin C cause MFM, while mutations in its actinbinding domain cause early-onset distal myopathy, preferentially affecting posterior leg compartment and hand muscles without MFM pathology. ${ }^{3-5}$ FLNC-MFM patients usually present only after the fourth decade of life. Respiratory muscles become affected with disease progression in $50 \%$ of patients. Cardiac abnormalities occur in one-third of patients, albeit mild. ${ }^{3}$ Symptomatic and supportive care, as well as cardiac and respiratory surveillance, are the mainstay treatment for patients with FLNC-MFM. Genetic counseling and the option of genetic testing should be offered to at-risk relatives.

\section{AUTHOR CONTRIBUTIONS}

Dr. Enduri: drafting the manuscript. Dr. Taylor: critical review of the manuscript. Dr. Liewluck: conceptualization and revising the manuscript.

\section{STUDY FUNDING}

No targeted funding reported.

\section{DISCLOSURE}

The authors report no disclosures relevant to the manuscript. Go to Neurology.org for full disclosures.

\section{REFERENCES}

1. Selcen D. Myofibrillar myopathies. Neuromuscul Disord 2011;21:161-171.

2. Kley RA, Hellenbroich Y, van der Ven PF, et al. Clinical and morphological phenotype of the filamin myopathy: a study of 31 German patients. Brain 2007;130:3250-3264.

3. Pfeffer G, Barresi R, Wilson IJ, et al. Titin founder mutation is a common cause of myofibrillar myopathy with early respiratory failure. J Neurol Neurosurg Psychiatry 2014;85: 331-338.

4. Kley RA, Serdaroglu-Oflazer P, Leber Y, et al. Pathophysiology of protein aggregation and extended phenotyping in filaminopathy. Brain 2012;135:2642-2660.

5. Duff RM, Tay V, Hackman P, et al. Mutations in the $\mathrm{N}$-terminal actin-binding domain of filamin C cause a distal myopathy. Am J Hum Genet 2011;88:729-740. 


\section{Neurology}

Clinical Reasoning: A 52-year-old woman with progressive proximal weakness Sailaja Enduri, Matthew R.G. Taylor and Teerin Liewluck

Neurology 2014;83;e106-e109

DOI 10.1212/WNL.0000000000000757

This information is current as of September 1, 2014

Updated Information \& Services

References

Subspecialty Collections

Permissions \& Licensing

Reprints including high resolution figures, can be found at: http://n.neurology.org/content/83/10/e106.full

This article cites 5 articles, 1 of which you can access for free at: http://n.neurology.org/content/83/10/e106.full\#ref-list-1

This article, along with others on similar topics, appears in the following collection(s):

Muscle disease

http://n.neurology.org/cgi/collection/muscle_disease

Information about reproducing this article in parts (figures,tables) or in its entirety can be found online at:

http://www.neurology.org/about/about_the_journal\#permissions

Information about ordering reprints can be found online:

http://n.neurology.org/subscribers/advertise

Neurology ${ }^{\circledR}$ is the official journal of the American Academy of Neurology. Published continuously since 1951, it is now a weekly with 48 issues per year. Copyright @ 2014 American Academy of Neurology. All rights reserved. Print ISSN: 0028-3878. Online ISSN: 1526-632X.

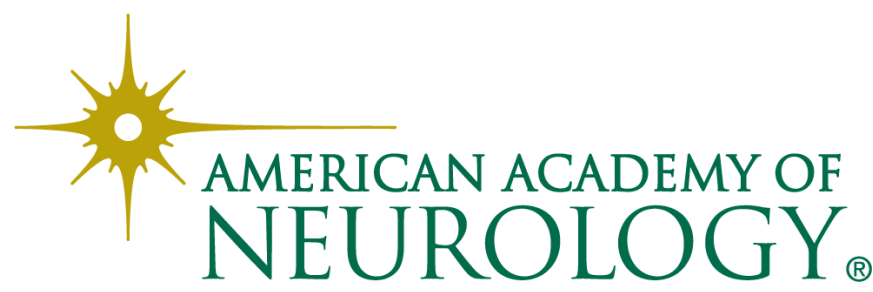

